\title{
General Fast Sampling Theorems for Nonlinear Systems
}

\author{
W. Bian and M. French \\ Department of Electronics and Computer Science, \\ University of Southampton, Southampton SO17 1BJ, UK \\ wb@ecs.soton.ac.uk, mcf@ecs.soton.ac.uk
}

\begin{abstract}
This paper is concerned with the gap metric approach to controller discretisation problems for continuous-time nonlinear systems with disturbances in both input and output channels. The principal idea is to construct a discrete controller based on a given stabilizing continuous time controller via a fast sampling and hold procedure and to calculate the gap between the two controllers. It is expected that, under general conditions, the computed gap depends on the discrete sample size and the faster the sample rate, the smaller the gap and, therefore, existing gap metric robust stability theorems can be applied to obtain both stability and performance results for the appropriately discretised controller. This is shown for the case of memoryless controllers and for a more general class of controllers specified by stable, causal operators. In both cases, both regional and global results are obtained under respective local and global incremental stability assumptions on the controllers.
\end{abstract}

Key words. Nonlinear feedback systems, digital controller, gap metric, stability

\section{Introduction}

Despite the prevalence of digital platforms for the implementation of modern control designs, the theory of nonlinear sampled data and discrete time systems has not been extensively developed: the majority of nonlinear controllers are synthesised in continuous time and then implemented via a strategy of fast sample and hold. Such a procedure is justified and well understood in the context of linear systems (see for example $[1,2,10]$ and the references therein); however there is relatively little theoretical justification for this approximation in the context of nonlinear controllers and plants.

The purpose of this paper is thus to provide rigorous fast sampling results which hold in a general i/o setting. When the underlying continuous-time closed loop system is known to be stable, we investigate the existence and estimation of a stabilizing sampling rate for corresponding sampled-data system based on sample and hold.

Most existing fast sampling results are for specific controller or system structures, however general settings in which the fast sampling and hold procedure has been justified can be found in $[5,11,13$, $14,15,16]$ and the references therein. In particular, general justifications for fast sampling are given in $[15,16]$ under the assumption of the existence of a quadratic Lyapunov function. In [11, 13, 14], sampled-data nonlinear systems (both with and without external disturbances) are considered within an ISS framework. The approach is via approximate discrete-time models, and explicit connections to the stability of the continuous-time plant model are made in [12]. The state-space results of $[11,12,13,14]$ yield semi-global practical stability of the closed loop.

The main results of this paper are obtained within an input/output framework and are closely related to $[11,12]$. Results of both a regional and a global nature are obtained, the latter results under more restrictive global conditions (but which, for example, recover the global stability properties for linear systems). All our results are obtained under either local or global incremental stability assumptions, which enable stability results (as opposed to the practical stability results if $[11,12]$ ) to 
be obtained. In particular this stronger form of stability enables a direct application of the robust stability results of [7] to the resulting sampled data closed loop, and consequently the gap metric robust stability margin is preserved asymptotically under suitably fast sampling. Furthermore, our results are in the context of i/o stability, and do not require e.g. the finite dimensional state space structures of $[11,12]$.

Discretisation of continuous-time controllers is clearly an approximation procedure, hence the aims of this paper can be viewed as developing conditions under which such fast sampling and holding controller approximations preserve both the stability and the performance of the original continuous time closed loop. By viewing the resulting discrete approximation of the continuous controller as a perturbation to the original controller, it is natural to address such questions within the framework of the nonlinear gap metric theory $[3,6,7,9]$. The principal idea is to construct a discrete controller based on a given stabilizing continuous time controller via a fast sampling and hold procedure and to calculate the gap between the two controllers.

We will consider two classes of controllers: the case of continuous, memoryless controllers and the more general case of controllers specified by stable, causal operators. In both cases, it is shown that, under general conditions, the computed gap depends on the discrete sample size such that the faster the sample rate, the smaller the gap and, therefore, existing gap metric robust stability theorems can be applied to obtain both stability and performance results for an appropriately discretised controller. In both cases, we require that the controllers are incrementally stable, and observe that many existing continuous time controllers lie within these categories. Unstable controllers are not considered in this paper; however, this class is clearly important and remains the subject of future work. However, we do note that the unstable case would appear to be substantially more technically involved.

The structure of the paper is as follows: Section 2 contains the background material on the gap metric and robust stability theorems. In Section 3, we give the definitions of discrete controller and obtain some of its basic properties. The main results on the stability and performance of the closed loop with the discretised controller corresponding to the case of a memoryless controller or a controller specified by a causal, stable operator are given in Sections 4 and 5 respectively.

\section{Preliminaries}

Let $\mathcal{U}, \mathcal{Y}$ represent normed input and output signal spaces and let $\mathcal{U}_{e}, \mathcal{Y}_{e}$ denote the auxiliary extended spaces, that is $\mathcal{U}_{e}=\left\{u: T_{\tau} u \in \mathcal{U}\right.$ for all $\left.\tau>0\right\}, \mathcal{Y}_{e}=\left\{y: T_{\tau} y \in \mathcal{Y}\right.$ for all $\left.\tau>0\right\}$, where $T_{\tau}$ is the truncation operator. Let $\mathcal{U}_{\omega}=\left\{u: T_{\tau} u \in \mathcal{U}\right.$ for all $\left.\tau \in(0, \omega)\right\}$, where $\omega \in(0, \infty]$, and let $\mathcal{U}_{a}=\cup_{\omega \in(0, \infty)} \mathcal{U}_{\omega} . \mathcal{Y}_{\omega}$ and $\mathcal{Y}_{a}$ are understood in the same manner. Plant operators will map from $\mathcal{U}_{a}$ to $\mathcal{Y}_{a}$ and controller operators will map from $\mathcal{Y}_{a}$ to $\mathcal{U}_{a}$. In the main context, we let $\mathcal{U}=L^{\infty}\left(\mathbb{R}_{+}, \mathbb{R}^{n}\right)=$ : $L_{n}^{\infty}\left(\mathbb{R}_{+}\right)$(or $\left.L_{n}^{\infty}\right)$, i.e. the set of all essentially bounded $n$-valued functions over $\mathbb{R}_{+}$, and let $\mathcal{Y}$ be a subspace of $L_{m}^{\infty}\left(\mathbb{R}_{+}\right)$. The space of all bounded $n$-valued ( $m$-valued, resp.) sequences is denoted by $l_{n}^{\infty}$ $\left(l_{m}^{\infty}\right.$, resp.). The space of all continuous $n$-valued signals is denoted by $C\left(\mathbb{R}_{+}, \mathbb{R}^{n}\right)$ and we also write $L^{\infty}=L_{1}^{\infty}, l^{\infty}=l_{1}^{\infty}$.

The Euclidean norm of $\mathbb{R}^{n}$ is denoted by $|\cdot|$ and that of a normed signal space, (say) $\mathcal{U}$, is denoted by $\|\cdot\|_{\mathcal{U}}$. The product space of $\mathcal{U}$ and $\mathcal{Y}$ is denoted by $\mathcal{W}$, i.e. $\mathcal{W}=\mathcal{U} \times \mathcal{Y}$ with norm

$$
\left\|\left(\begin{array}{l}
u \\
y
\end{array}\right)\right\|_{\mathcal{W}}=\left\|(u, y)^{\top}\right\|_{\mathcal{W}}=\max \left\{\|u\|_{\mathcal{U}},\|y\|_{\mathcal{Y}}\right\} .
$$

The closed disc in $\mathcal{W}$ centred at 0 with radius $r$ is denoted by $\mathcal{S}_{r}$. We also let $\mathcal{W}_{a}=\mathcal{U}_{a} \times \mathcal{Y}_{a}, \mathcal{W}_{e}=$ $\mathcal{U}_{e} \times \mathcal{Y}_{e}$ and let $\mathcal{K}_{\infty}$ be the set of all continuous increasing function $g:[0, \infty) \rightarrow[0, \infty)$ such that $g(0)=0, g(\infty)=\infty$.

Given a signal operator $H: \mathcal{U}_{a} \rightarrow \mathcal{Y}_{a}$, we define a domain and graph, respectively, as follows:

$$
\operatorname{Dom}(H)=\{u \in \mathcal{U}: H u \in \mathcal{Y}\}, \quad \mathcal{G}_{H}=\left\{(y, H y)^{\top}: u \in \operatorname{Dom}(H)\right\}
$$


and let

$$
\begin{aligned}
& \|H\|:=\sup \left\{\frac{\|H u\|_{\mathcal{Y}}}{\|u\|_{\mathcal{U}}}: u \in \mathcal{G}_{H} \text { with }\|u\|_{\mathcal{U}} \neq 0\right\}, \\
& \gamma[H](r):=\sup \left\{\|H u\|_{\mathcal{Y}}: u \in \mathcal{G}_{H} \text { with }\|u\|_{\mathcal{U}} \leq r\right\} \text { for } r \geq 0, \\
& \|H\|_{\triangle}:=\sup \left\{\frac{\left\|H u_{1}-H u_{2}\right\|_{\mathcal{Y}}}{\left\|u_{1}-u_{2}\right\|_{\mathcal{U}}}: u_{1}, u_{2} \in \mathcal{G}_{H}\right\} .
\end{aligned}
$$

Definition 2.1. A signal operator $H$ is said to be: (i) gain stable if $\|H\|<\infty$; (ii) gf-stable (gain function stable) if $\gamma[H](r)<\infty$ for each $r \geq 0$; and (iii) incrementally stable if $\|H\|_{\triangle}<\infty$.

Obviously a necessary condition for gain stability is $H u \rightarrow 0$ as $u \rightarrow 0$. Hence, throughout this paper, every signal operator is assumed to have an equilibrium at 0 , that is, $H(0)=0$.

We now consider the control system $[P, C]$ described by the standard configuration Figure 1 . Here

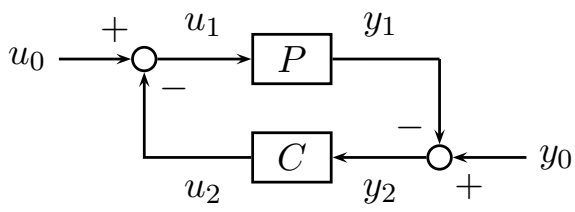

Figure 1: Standard Feedback Configuration.

$u_{i} \in \mathcal{U}_{a}, y_{i} \in \mathcal{Y}_{a}$ for $i=0,1,2$, and both the plant $P: \mathcal{U}_{a} \rightarrow \mathcal{Y}_{a}$ and compensator $C: \mathcal{Y}_{a} \rightarrow \mathcal{U}_{a}$ are causal and in general nonlinear.

Definition 2.2. If, for each $\left(u_{0}, y_{0}\right) \in \mathcal{W}$, there exist unique signals $u_{1}, u_{2} \in \mathcal{U}_{a}$ and $y_{1}, y_{2} \in \mathcal{Y}_{a}$ such that

$$
u_{0}=u_{1}+u_{2}, \quad y_{0}=y_{1}+y_{2}, \quad y_{1}=P u_{1}, \quad u_{2}=C y_{2}
$$

and the feedback operator

$$
H_{P, C}: \mathcal{W} \rightarrow \mathcal{W}_{e} \times \mathcal{W}_{e}:\left(\begin{array}{l}
u_{0} \\
y_{0}
\end{array}\right) \mapsto\left(\left(\begin{array}{l}
u_{1} \\
y_{1}
\end{array}\right),\left(\begin{array}{l}
u_{2} \\
y_{2}
\end{array}\right)\right)
$$

is defined and causal, then the closed loop $[P, C]$ is said to be globally well-posed. If $\mathcal{D} \subset \mathcal{W}$ and $\left.H_{P, C}\right|_{\mathcal{D}}$ is defined and causal, then $[P, C]$ is said to be globally well posed on $\mathcal{D}$.

A further weaker notion of well-posedness is also introduced as in [7], namely

Definition 2.3. The system is said locally well-posed if, for each $\left(u_{0}, y_{0}\right) \in \mathcal{W}$, there exist unique signals $u_{1}, u_{2} \in \mathcal{U}_{a}$ and $y_{1}, y_{2} \in \mathcal{Y}_{a}$ satisfying (2.1) over a maximal interval $[0, \omega)$ with $0<\omega \leq \infty$, $H_{P, C}: \mathcal{W} \rightarrow \mathcal{W}_{a} \times \mathcal{W}_{a}$ is causal on $[0, \omega)$ and, if $\omega<\infty$, then $\left\|\left(u_{1}, y_{1}\right)^{\top}\right\|_{L^{\infty}[0, t)} \rightarrow \infty$ as $t \rightarrow \omega$ from below.

The stability of a closed loop system $[P, C]$ is determined by the stability of the corresponding operator $H_{P, C}$ :

Definition 2.4. A well-posed closed loop $[P, C]$ is said to be gain stable (resp. gf-stable, incrementally stable) if $H_{P, C}$ is stable in the same sense. 
It is well known that stability of $[P, C]$ is equivalent to the same notion of stability of either of the following mappings:

$$
\Pi_{P / / C}:\left(\begin{array}{c}
u_{0} \\
y_{0}
\end{array}\right) \mapsto\left(\begin{array}{c}
u_{1} \\
y_{1}
\end{array}\right), \quad \Pi_{C / / P}:\left(\begin{array}{c}
u_{0} \\
y_{0}
\end{array}\right) \mapsto\left(\begin{array}{c}
u_{2} \\
y_{2}
\end{array}\right)
$$

There are a number of generalizations of the gap metric concept for nonlinear systems, see eg. $[3,7,9]$. Here we recall the gap metric defined in [7] by Georgiou and Smith. That is, for two signal operators $H, H_{1}$, we define the directed gap as follows:

$$
\vec{\delta}\left(H, H_{1}\right):= \begin{cases}\inf _{\Phi \in \mathcal{O}}\|I-\Phi\| & \text { if } \mathcal{O} \neq \emptyset \\ \infty & \text { if } \mathcal{O}=\emptyset\end{cases}
$$

where

$$
\mathcal{O}=\left\{\Phi: \mathcal{G}_{H} \rightarrow \mathcal{G}_{H_{1}} \mid \Phi 0=0, \Phi \text { is causal, surjective }\right\} .
$$

Using this definition Georgiou and Smith in [7] studied the robustness of nonlinear systems. Within the language of this paper, the following results follow from [7]:

Theorem 2.5. Suppose $[P, C]$ is globally well posed and $\left[P, C_{1}\right]$ is locally well-posed. and

(i) If $[P, C]$ is gain stable and $\vec{\delta}\left(C, C_{1}\right)<\left\|\Pi_{C / / P}\right\|^{-1}$, then $\left[P, C_{1}\right]$ is globally well posed, gain stable

$$
\left\|\Pi_{C_{1} / / P}\right\| \leq\left\|\Pi_{C / / P}\right\| \frac{1+\vec{\delta}\left(C, C_{1}\right)}{1-\vec{\delta}\left(C, C_{1}\right)\left\|\Pi_{C / / P}\right\|} .
$$

(ii) If $[P, C]$ is gf-stable, there exists a surjective mapping $\Phi: \mathcal{D} \rightarrow \mathcal{G}_{C_{1}}$ where $\mathcal{D} \subset \mathcal{G}_{C}$, and there exists a function $\varepsilon(\cdot) \in \mathcal{K}_{\infty}$ such that $\gamma[I-\Phi] \circ \gamma\left[\Pi_{C / / P}\right](r) \leq(1+\varepsilon)^{-1}(r)$ for all $r \geq 0$, then $\left[P, C_{1}\right]$ is globally well posed and gf-stable.

Theorem 2.6. Let $r>0$. Suppose $[P, C]$ is globally well-posed on $\mathcal{S}_{r},\left[P, C_{1}\right]$ is locally well-posed and $\left\|\left.\Pi_{C / / P}\right|_{\mathcal{S}_{r}}\right\| \leq p$. Suppose that there exists a mapping $\Phi: \mathcal{G}_{C} \cap \mathcal{S}_{p r} \rightarrow \mathcal{G}_{C_{1}}$ such that $\left\|\left.(\Phi-I)\right|_{\mathcal{G}_{C} \cap \mathcal{S}_{\text {pr }}}\right\|=$ $q<1 / p$ and $\left.(\Phi-I) \Pi_{C / / P}\right|_{\mathcal{S}_{r}}$ is continuous and compact. Then $\left[P, C_{1}\right]$ is globally well-posed and bounded on $\mathcal{S}_{(1-p q) r}$ and

$$
\left\|\Pi_{C_{1} / / P}\left|\mathcal{S}_{(1-p q) r}\|\leq\| \Pi_{C / / P}\right|_{\mathcal{S}_{r}}\right\| \frac{1+q}{1-q\left\|\Pi_{C / / P} \mid \mathcal{S}_{r}\right\|}
$$

\section{The Digital Controllers}

In this section, we construct a discrete-time controller $C_{d}$ which, under the sample and hold operation, approximates a given continuous-time controller $C$, with the additional property that the stability of $[P, C]$ is preserved when the sample and hold implementation of $C_{d}$ is utilized in place of $C$. The construction is a standard approach and is described next.

We first introduce the concrete signal space setting in which we will present our results. Let $\alpha>0, \tau>0$ be given and define normed input and output spaces as:

$$
\begin{aligned}
& \mathcal{U}=\left\{u \in L^{\infty}\left(\mathbb{R}_{+}, \mathbb{R}^{n}\right): \sup _{t \geq 0}|u(t)|<\infty\right\} \\
& \mathcal{Y}_{\alpha, \tau}=\left\{\begin{aligned}
y & \text { is continuous on each interval }[k \tau,(k+1) \tau), \\
y \in L^{\infty}\left(\mathbb{R}_{+}, \mathbb{R}^{m}\right): & k \in \mathbb{N} \text { and } \sup _{\substack{t, s \in[k \tau,(k+1) \tau) \\
k \in \mathbb{N}, t \neq s}} \frac{|y(t)-y(s)|}{|t-s|^{\alpha}}<\infty
\end{aligned}\right\} .
\end{aligned}
$$


where

$$
\|u\|_{\mathcal{U}}=\sup _{t \geq 0}|u(t)|, \quad\|y\|_{\mathcal{Y}_{\alpha, \tau}}=\max \left\{\|y\|_{L_{m}^{\infty},} \sup _{\substack{t, s \in[k \tau,(k+1) \tau) \\ k \in \mathbb{N}, t \neq s}} \frac{|y(t)-y(s)|}{|t-s|^{\alpha}}\right\} .
$$

The signal space $\mathcal{Y}_{\alpha, \tau}$ is the set of all piecewise regular signals; we note that similar regular signal spaces have been exploited in [8] for the analysis of the robustness of a relaxation oscillator. For notational simplicity we sometimes write $\mathcal{Y}=\mathcal{Y}_{\alpha, \tau}, \mathcal{Y}_{e}=\left(\mathcal{Y}_{\alpha, \tau}\right)_{e}, \mathcal{Y}_{a}=\left(\mathcal{Y}_{\alpha, \tau}\right)_{a}$.

Since the input and output of the controller are both continuous-time signals, two further operations are needed as shown in Figure 2 below. $S_{\tau}$ denotes a sample operator which discretises the input continuous-time signal so that the discrete-time controller $C_{d}$ can be applied. A second operator (the hold operator) $H_{\tau}$ transforms the discrete signal-time back to piecewise continuous-time signal for the system $P$ to recognize. Here, $\tau$ represents the step size of the sample and hold operation. The sample

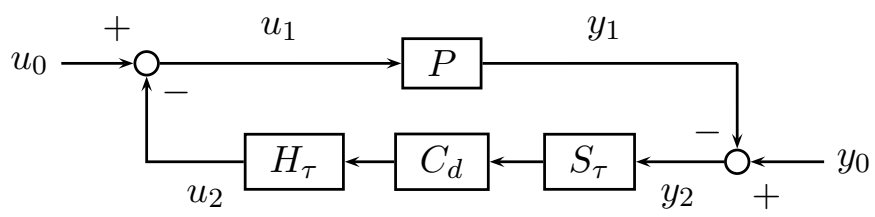

Figure 2: Standard Feedback Configuration.

operator, $S_{\tau}$, is naturally chosen to be:

$$
S_{\tau}: \mathcal{Y}_{\alpha, \tau} \rightarrow l_{m}^{\infty}, \quad S_{\tau} y=z=\left(z_{k}\right) \quad \text { with } z_{k}=y(k \tau), k=0,1,2, \cdots \text { for any } y \in \mathcal{Y}_{\alpha, \tau} .
$$

There are several choices for $H_{\tau}$. Here we consider the "ZOH operator":

$$
H_{\tau}: l_{n}^{\infty} \rightarrow \mathcal{U}, \quad\left(H_{\tau} z\right)(t)=z_{k} \text { for } t \in[k \tau,(k+1) \tau) \quad \text { for any } z=\left(z_{k}\right) \in l_{n}^{\infty} .
$$

The controller $C_{d}: l_{m}^{\infty} \rightarrow l_{n}^{\infty}$ depends on a known stable continuous-time controller $C: \mathcal{Y}_{\alpha, \tau} \rightarrow \mathcal{U}$ via a process of sampling. We consider the cases where $C$ is either governed by a memoryless function or is a stable operator between signal spaces. If $C$ is memoryless, i.e. there exists a memoryless function $K: \mathbb{R}^{m} \rightarrow \mathbb{R}^{n}$ such that $C: u_{2}(t)=K\left(y_{2}(t)\right)$, then we let

$$
C_{d} z=\left\{K\left(z_{k}\right)\right\}_{k \geq 0}, \quad \text { for all } z=\left\{z_{k}\right\}_{k \geq 0} \in l_{m}^{\infty} .
$$

If $C$ is given by a stable causal operator, we let

$$
C_{d} z=S_{\tau} C H_{\tau} z \quad \text { for all } z \in l_{m}^{\infty} .
$$

Note that (3.5) is a generalization of (3.4). We notice that since $C$ is stable, $(C y)(t)$ is defined for every piecewise continuous function $y$ and for all $t \geq 0$ thus ensuring $C_{d}$ and $H_{\tau} C_{\tau}$ are defined in both cases in (3.4) and (3.5). Under such assumptions, $C_{d}$ is always a causal operator from $l_{m}^{\infty}$ to $l_{n}^{\infty}$.

Let $C_{\tau}: \mathcal{Y}_{\alpha, \tau} \rightarrow \mathcal{U}$ be defined:

$$
C_{\tau}=H_{\tau} C_{d} S_{\tau}=H_{\tau} S_{\tau} C H_{\tau} S_{\tau}
$$

and note this maps signals in $\mathcal{Y}_{\alpha, \tau}$ to step functions. To study the properties of $C_{\tau}$, we first consider the operator $H_{\tau} S_{\tau}$. From the definitions, it follows that

$$
\left\|H_{\tau} S_{\tau} y\right\|_{L_{m}^{\infty}} \leq\|y\|_{L_{m}^{\infty}} \quad \text { and } \quad\left\|H_{\tau} S_{\tau} x-H_{\tau} S_{\tau} y\right\|_{L_{m}^{\infty}} \leq\|x-y\|_{L_{m}^{\infty}}
$$


for any piecewise continuous functions $x, y \in L_{m}^{\infty}$. In particular, $H_{\tau} S_{\tau} c=c$ for constant function $c$. Consequently, if understand $H_{\tau} S_{\tau}$ as an operator from either $L_{m}^{\infty}$ or $\mathcal{Y}_{\alpha, \tau}$ to $L_{m}^{\infty}$, we have

$$
\left\|H_{\tau} S_{\tau}\right\|=1 \quad \text { for each } \tau \text {. }
$$

For any $y \in \mathcal{Y}_{\alpha, \tau}$ and $k \in \mathbb{N}$, by the definitions of $\|\cdot\|_{\mathcal{Y}}$, we also have

$$
\begin{aligned}
& \|y-y(k \tau)\|_{L_{m}^{\infty}[k \tau,(k+1) \tau)} \leq \tau^{\alpha}\|y\|_{\mathcal{Y}} \\
& \left\|y-H_{\tau} S_{\tau} y\right\|_{L_{m}^{\infty}} \leq \tau^{\alpha}\|y\|_{\mathcal{Y}} .
\end{aligned}
$$

Proposition 3.1. (i) If $C$ is gain stable (resp., gf-stable), then $C_{\tau}$ is gain stable (resp., gf-stable);

(ii) If $C$ is incrementally stable, then $C_{\tau}$ is incrementally stable and $\left\|C_{\tau}\right\|_{\triangle}=\|C\|_{\triangle}$;

(iii) If $\left.C\right|_{\mathcal{S}_{r}}$ is incrementally stable, then $\left.C_{\tau}\right|_{\mathcal{S}_{r}}$ is incrementally stable and $\left\|\left.C_{\tau}\right|_{\mathcal{S}_{r}}\right\|_{\triangle}=\left\|\left.C\right|_{\mathcal{S}_{r}}\right\|_{\triangle}$.

Proof: (i) Since $C_{\tau}=H_{\tau} S_{\tau} C H_{\tau} S_{\tau}$, the conclusion follows from (3.7).

(ii) Suppose $x, y \in \mathcal{Y}_{\alpha, \tau}$ and $C$ is incrementally stable. Then, by (3.6), we have

$$
\begin{aligned}
\left\|C_{\tau} y-C_{\tau} x\right\|_{\mathcal{U}} & =\left\|H_{\tau} S_{\tau} C H_{\tau} S_{\tau} y-H_{\tau} S_{\tau} C H_{\tau} S_{\tau} x\right\|_{\mathcal{U}} \\
& \leq\left\|C\left(H_{\tau} S_{\tau} y\right)-C\left(H_{\tau} S_{\tau} x\right)\right\|_{\mathcal{U}} \leq\|C\|_{\triangle}\|y-x\|_{L_{m}^{\infty}} \leq\|C\|_{\triangle}\|y-x\|_{\mathcal{Y}_{\alpha}} .
\end{aligned}
$$

So $C_{\tau}$ is incrementally stable and $\left\|C_{\tau}\right\|_{\triangle} \leq\|C\|_{\triangle}$. Since $C_{\tau} y=C y$ for $y(t) \equiv c$ (constant), we also see that $\left\|C_{\tau}\right\|_{\triangle} \geq\|C\|_{\triangle}$.

(iii) Similar to (ii).

\section{Closed Loop Stability with a Memoryless Controller}

In this section, we study the stability of the closed loop $\left[P, C_{\tau}\right]$ in terms of the stability of $[P, C]$ in the case where $C$ is memoryless. Our results show that the sampled data controller $C_{\tau}$ stabilizes the nominal plant $P$ provided $[P, C]$ is stable in the same sense and $\tau$ is sufficiently small.

The approach is to consider $C_{\tau}$ to be a perturbation to $C$ and exploit the gap metric theory of [7]. To apply the results in [7], we need to construct a surjective mapping $\Phi$ from $\mathcal{G}_{C}$ to $\mathcal{G}_{C_{\tau}}$, see $(2.3)$ and (2.4). Throughout this paper, we consider the natural surjective mapping

$$
\Phi_{\tau}: \mathcal{G}_{C} \rightarrow \mathcal{G}_{C_{\tau}}, \quad \Phi_{\tau}\left(\begin{array}{c}
y_{2} \\
C y_{2}
\end{array}\right)=\left(\begin{array}{c}
y_{2} \\
C_{\tau} y_{2}
\end{array}\right)
$$

Lemma 4.1. Let $\tau>0$ and suppose $C: \mathcal{Y}_{\alpha, \tau} \rightarrow \mathcal{U}$ be memoryless. Then:

(i) If $C$ is incrementally stable then $\left\|I-\Phi_{\tau}\right\| \leq \tau^{\alpha}\|C\|_{\triangle}$ and therefore $\vec{\delta}\left(C, C_{\tau}\right) \leq \tau^{\alpha}\|C\|_{\triangle}$.

(ii) If $\left.C\right|_{\mathcal{S}_{r}}$ is incrementally stable then $\left\|\left.\left(I-\Phi_{\tau}\right)\right|_{\mathcal{S}_{r}}\right\| \leq \tau^{\alpha}\left\|\left.C\right|_{\mathcal{S}_{r}}\right\|_{\triangle}$.

(iii) If there exist a non-decreasing function $h: \mathbb{R}_{+} \rightarrow \mathbb{R}_{+}$such that

$$
|C(y(t))-C(y(s))| \leq h(\max \{|y(t)|,|y(s)|\})|y(t)-y(s)|, \text { for each } y \in \mathcal{Y}_{\alpha, \tau}, t, s \in \mathbb{R}_{+}
$$

then $\gamma\left[I-\Phi_{\tau}\right](r) \leq r h(r) \tau^{\alpha}$.

Proof: Let $\tau>0$ and suppose $C: \mathcal{Y}_{\alpha, \tau} \rightarrow \mathcal{U}$ is memoryless i.e. $C\left(y_{2}\right)(t)=K\left(y_{2}(t)\right)$ where $K: \mathbb{R}^{m} \rightarrow$ $\mathbb{R}^{n}$. 
(i) Since $C_{\tau} y_{2}=H_{\tau} C_{d} S_{\tau} y_{2}$, we have

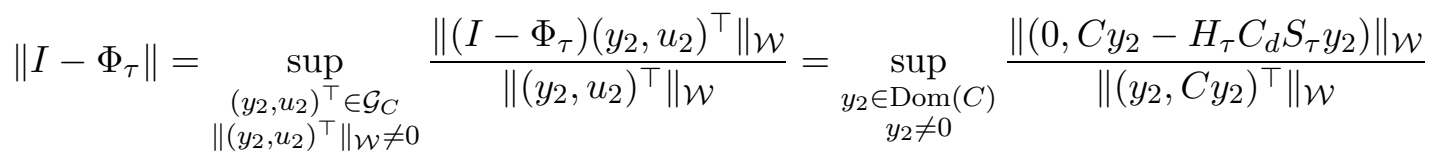

$$
\begin{aligned}
& \leq \sup _{\substack{y_{2} \in \operatorname{Dom}(C) \\
y_{2} \neq 0}} \frac{\left\|C y_{2}-H_{\tau} C_{d} S_{\tau} y_{2}\right\|_{\mathcal{U}}}{\max \left\{\left\|y_{2}\right\| \mathcal{Y},\left\|C y_{2}\right\|_{\mathcal{U}}\right\}}
\end{aligned}
$$

By the incremental stability of $C$ and (3.8), we obtain

$$
\begin{aligned}
\left\|C y_{2}-H_{\tau} C_{d} S_{\tau} y_{2}\right\|_{\mathcal{U}} & \left.\left.=\sup _{\substack{t \in[k \tau,(k+1) \tau) \\
k>0}} \mid C\left(y_{2}\right)(t)\right)-\left(H_{\tau} C S_{\tau} y_{2}\right)(t)\right)\left|=\sup _{\substack{t \in[k \tau,(k+1) \tau) \\
k>0}}\right| K\left(y_{2}(t)\right)-K\left(y_{2}(k \tau)\right) \mid \\
& \leq\|C\|_{\triangle} \sup _{\substack{t \in[k \tau,(k+1) \tau) \\
k>0}}\left|y_{2}(t)-y_{2}(k \tau)\right| \leq \tau^{\alpha}\|C\|_{\triangle}\left\|y_{2}\right\| \mathcal{Y}
\end{aligned}
$$

Hence $\left\|I-\Phi_{\tau}\right\| \leq \tau^{\alpha}\|C\|_{\triangle}$ and, therefore, $\vec{\delta}\left(C, C_{\tau}\right) \leq \tau^{\alpha}\|C\|_{\triangle}$.

(ii) Similar to (i).

(iii) Suppose there exists a non-decreasing function $h: \mathbb{R} \rightarrow \mathbb{R}_{+}$such that (4.2) holds. Then for each $r>0$,

$$
\begin{aligned}
\gamma\left(I-\Phi_{\tau}\right)(r)= & \sup _{\substack{\left(y_{2}, C y_{2}\right)^{\top} \in \mathcal{G}_{C} \\
\left\|\left(y_{2}, C y_{2}\right)^{\top}\right\|_{\mathcal{W}} \leq \alpha}} \sup _{t \in[k \tau,(k+1) \tau)}\left|C\left(y_{2}(t)\right)-C\left(y_{2}(k \tau)\right)\right| \\
\leq & \sup _{\substack{\left(y_{2}, C y_{2}\right)^{\top} \in \mathcal{G}_{C} \\
\left\|\left(y_{2}, C y_{2}\right)^{\top}\right\|_{\mathcal{W}} \leq \alpha}} \sup _{t \in[k \tau,(k+1) \tau)} h\left(\max \left\{\left|y_{2}(t)\right|,\left|y_{2}(k \tau)\right|\right\}\right)\left|y_{2}(t)-y_{2}(k \tau)\right| \\
\leq & \sup _{\substack{\left(y_{2}, C y_{2}\right)^{\top} \in \mathcal{G}_{C}\\
}} h\left(\left\|y_{2}\right\|_{\mathcal{Y}}\right)\left\|y_{2}\right\| \mathcal{Y} \leq r h(r) \tau^{\alpha}, \\
& \left\|\left(y_{2}, C y_{2}\right)^{\top}\right\|_{\mathcal{W} \leq \alpha} \leq
\end{aligned}
$$

as required.

We remark that when $C$ is Lipschitz or incrementally stable, (4.2) is satisfied with $h(r) \equiv\|C\|_{\triangle}$. Suitable functions $h(\cdot)$ also exist for operators with nonlinear growth, eg. if $K(y)=-y^{2}$, then $h(r)=2 r$.

Hence, by Theorem 2.5, we obtain the following global result:

Theorem 4.2. Let $\tau>0$ and let $\mathcal{U}, \mathcal{Y}_{\alpha, \tau}$ be defined as in (3.1)-(3.3). Suppose that $P: \mathcal{U}_{a} \rightarrow\left(\mathcal{Y}_{\alpha, \tau}\right)_{a}$ is causal, $C:\left(\mathcal{Y}_{\alpha, \tau}\right)_{a} \rightarrow \mathcal{U}_{a}$ is memoryless, $[P, C]$ is globally well-posed and $\left[P, C_{\tau}\right]$ is locally well-posed. (i) If $[P, C]$ is gain stable, $C$ is incrementally stable and $\tau^{\alpha}<\left\|\Pi_{C / / P}\right\|^{-1}\|C\|_{\triangle}^{-1}$, then $\left[P, C_{\tau}\right]$ is globally well-posed, gain stable and

$$
\left\|\Pi_{C_{\tau} / / P}\right\| \leq\left\|\Pi_{C / / P}\right\| \frac{1+\tau^{\alpha}\|C\|_{\triangle}}{1-\tau^{\alpha}\|C\|_{\triangle}\left\|\Pi_{C / / P}\right\|} .
$$

(ii) If $[P, C]$ is gf-stable, inequality (4.2) is satisfied and there exists $\varepsilon \in \mathcal{K}_{\infty}$ such that

$$
\tau^{\alpha} \leq \frac{(1+\varepsilon)^{-1}(r)}{h\left(\gamma\left[\Pi_{C / / P}\right](r)\right) \gamma\left[\Pi_{C / / P}\right](r)} \quad \text { for all } r \geq 0,
$$

then $\left[P, C_{\tau}\right]$ is globally well-posed and gf-stable. 
We remark that Theorem 4.2 part (ii) may yield sharper estimates for the stabilizing sample rate than part (i).

By Theorem 2.6, we have the following regional stability theorem:

Theorem 4.3. Let $r, \tau>0$ and let $\mathcal{U}, \mathcal{Y}_{\alpha, \tau}$ be defined as in (3.1)-(3.3). Suppose that $P: \mathcal{U}_{a} \rightarrow\left(\mathcal{Y}_{\alpha, \tau}\right)_{a}$ is causal, $C:\left(\mathcal{Y}_{\alpha, \tau}\right)_{a} \rightarrow \mathcal{U}_{a}$ is memoryless, $[P, C]$ is globally well-posed on $\mathcal{S}_{r}$ and $\left[P, C_{\tau}\right]$ is locally wellposed. Suppose $\left\|\Pi_{C / / P} \mid \mathcal{S}_{r}\right\| \leq p$ and that the mapping $\Phi_{\tau}$ defined in (4.1) is such that $\left(\Phi_{\tau}-I\right) \Pi_{C / / P} \mid \mathcal{S}_{r}$ is continuous and compact. If $q=\tau^{\alpha}\left\|\left.C\right|_{\mathcal{S}_{r}}\right\|_{\triangle}<p^{-1}$, then $\left[P, C_{\tau}\right]$ is globally well-posed and bounded on $\mathcal{S}_{(1-p q) r}$, and

$$
\left\|\left.\Pi_{C_{\tau} / / P}\right|_{\mathcal{S}_{(1-p q) r}}\right\| \leq\left\|\left.\Pi_{C / / P}\right|_{\mathcal{S}_{r}}\right\| \frac{1+\tau^{\alpha}\left\|\left.C\right|_{\mathcal{S}_{r}}\right\|_{\triangle}}{1-\tau^{\alpha}\left\|\left.C\right|_{\mathcal{S}_{r}}\right\| \Delta\left\|\Pi_{C / / P} \mid \mathcal{S}_{r}\right\|}
$$

Next, we present some examples to show the applications of this theorem.

Example 4.4. (Gain stability of an integrator with saturation) We let $n=m=\alpha=1$ and consider the feedback system given in Figure 3.

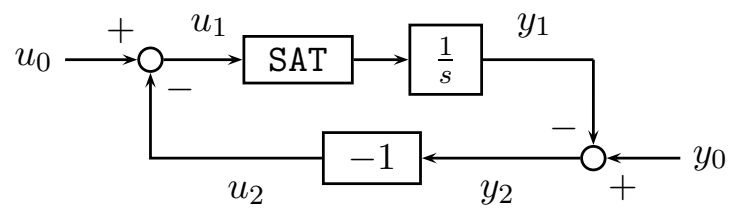

Figure 3: Integrator with saturation.

In this system, the nominal plant $P$ is defined by

$$
\begin{aligned}
& \dot{x}(t)=\operatorname{SAT}\left(u_{1}(t)\right), \quad x(0)=0 \\
& y_{1}(t)=x(t)
\end{aligned}
$$

where $\operatorname{SAT}\left(u_{1}\right)=u_{1}$ when $\left|u_{1}\right| \leq 1$ and is equal to $\operatorname{sign}\left(u_{1}\right)$ when $\left|u_{1}\right|>1$. The memoryless controller $C$ is specified by $K\left(y_{2}\right)=-y_{2}$.

It is routine to check that $P(\mathcal{U}) \subset \mathcal{Y}, C: \mathcal{Y} \rightarrow \mathcal{U}$ is incrementally stable with $\|C\|_{\triangle}=1$ and that

$$
\left\|\Pi_{C / / P}\right\|=\sup _{\left\|\left(u_{0}, y_{0}\right)^{\top}\right\| \neq 0} \frac{\left\|y_{0}-x\right\|_{\mathcal{Y}}}{\max \left\{\left\|u_{0}\right\|_{\mathcal{U}},\left\|y_{0}\right\|_{\mathcal{Y}}\right\}} \leq \sup _{\left\|\left(u_{0}, y_{0}\right)^{\top}\right\| \neq 0} \frac{\left\|y_{0}\right\|_{\mathcal{Y}}+\|x\|_{\mathcal{Y}}}{\max \left\{\left\|u_{0}\right\|_{\mathcal{U}},\left\|y_{0}\right\|_{\mathcal{Y}}\right\}},
$$

where $x$ is the solution to the closed loop equation

$$
\dot{x}(t)=\operatorname{SAT}\left(u_{0}(t)+y_{0}(t)-x(t)\right), \quad x(0)=0 .
$$

For a given $u_{0}, y_{0}$, consider the corresponding continuous function $x(\cdot)$ over any finite given interval $[0, T]$. Suppose the maximum of $|x(t)|$ on $[0, T]$ is reached at $t_{0}$ and $x\left(t_{0}\right)>0$. Then, for any $\varepsilon>0$, there exist $t_{1} \in\left(0, t_{0}\right)$ such that $x\left(t_{1}\right)>x\left(t_{0}\right)-\varepsilon$ and $\dot{x}(t)>0$. From (4.3), it follows $u_{0}\left(t_{1}\right)+y_{0}\left(t_{1}\right)>x\left(t_{1}\right)>x\left(t_{0}\right)-\varepsilon=\|x\|_{L^{\infty}(0, T)}-\varepsilon$. A similar argument when $x\left(t_{0}\right)<0$ establishes $\left\|u_{0}+y_{0}\right\|_{L^{\infty}} \geq\|x\|_{L^{\infty}}$. By (4.3) we have

$$
|x(t)-x(s)| \leq \sup _{r>0}|\dot{x}(r)||t-s| \leq \sup _{r>0}\left|\operatorname{SAT}\left(u_{0}(r)+y_{0}(r)-x(r)\right)\right||t-s| \leq\left\|u_{0}+y_{0}-x\right\|_{L^{\infty}}|t-s| .
$$

Hence $\|x\|_{\mathcal{Y}} \leq \max \left\{\|x\|_{L^{\infty}},\left\|u_{0}+y_{0}-x\right\|_{L^{\infty}}\right\} \leq 2\left\|u_{0}+y_{0}\right\|_{L^{\infty}}$ and therefore

$$
\left\|\Pi_{C / / P}\right\| \leq \sup _{\left\|\left(u_{0}, y_{0}\right)^{\top}\right\| \neq 0} \frac{\left\|y_{0}\right\| \mathcal{Y}+2\left\|u_{0}+y_{0}\right\|_{L^{\infty}}}{\max \left\{\left\|u_{0}\right\| \mathcal{U},\left\|y_{0}\right\|_{\mathcal{Y}}\right\}} \leq \sup _{\left\|\left(u_{0}, y_{0}\right)^{\top}\right\| \neq 0} \frac{2\left\|u_{0}\right\|_{\mathcal{U}}+3\left\|y_{0}\right\|_{\mathcal{Y}}}{\max \left\{\left\|u_{0}\right\| \mathcal{U},\left\|y_{0}\right\|_{\mathcal{Y}}\right\}} \leq 5 .
$$


So $[P, C]$ is globally well-posed and gain stable. Since $\left[P, C_{\tau}\right]$ is locally well-posed by standard result on differential systems, it follows by Lemma 2.5 and Theorem 4.2 that $\left[P, C_{\tau}\right]$ is gain stable provided $\tau<1 / 5$.

Example 4.5. (A gain stable system with cubic nonlineaity) Let $m=n=\alpha=1$ as in Example 4.4 and consider the system where the nominal plant $P$ is defined by

$$
\dot{x}(t)=-x^{3}(t)+u_{1}(t), y_{1}(t)=x(t), \quad x(0)=0,
$$

and the controller $C$ is defined by $u_{2}(t)=-y_{2}(t)$.

For this system, the closed loop is $[P, C]$ given by

$$
\dot{x}(t)=-x^{3}(t)-x(t)+u_{0}(t)+y_{0}(t), y_{1}(t)=x(t), \quad x(0)=0
$$

and the parallel projection $\Pi_{C / / P}$ is the mapping $\left(u_{0}, y_{0}\right)^{\top} \mapsto\left(-y_{0}+x, y_{0}-x\right)^{\top}$. From (4.5), it follows $x(t) \dot{x}(t) \leq-|x(t)|^{4}-|x(t)|^{2}+\left|u_{0}(t)+y_{0}(t)\right||x(t)|$ and, therefore $|x(t)|^{3}+|x(t)| \leq\left|u_{0}(t)+y_{0}(t)\right| \leq$ $\left\|u_{0}+y_{0}\right\|_{L^{\infty}}$ for all $t>0$. This yields

$$
\sup _{t \neq s} \frac{|x(t)-x(s)|}{|t-s|} \leq \max _{t \geq 0}|\dot{x}(t)| \leq \max _{t \geq 0}\left(|x(t)|^{3}+|x(t)|+\mid u_{0}(t)+y_{0}(t) \|\right) \leq 4\left\|\left(u_{0}, y_{0}\right)^{\top}\right\| .
$$

So $\|x\|_{\mathcal{Y}} \leq 4\left\|\left(u_{0}, y_{0}\right)^{\top}\right\|$ and $\left\|y_{0}-x\right\|_{\mathcal{Y}} \leq 5\left\|\left(u_{0}, y_{0}\right)^{\top}\right\|$. That, is $[P, C]$ is gain stable and $\left\|\Pi_{C / / P}\right\| \leq 5$. Hence, by Theorem $4.2,\left[P, C_{\tau}\right]$ is gain stable provided $\tau<1 / 5$.

\section{Closed Loop Stability with a Dynamic Controller}

We now consider the case where the controller is dynamic. In particular, we restrict our attention to the case where $C$ is given by a stable causal operator. In this context, a condition stronger then (4.2) is utilized to estimate $\left\|I-\Phi_{\tau}\right\|$.

Lemma 5.1. Let $\tau>0$ and suppose that $C: \mathcal{Y}_{\alpha, \tau} \rightarrow \mathcal{U}$ is causal. Let $\Phi_{\tau}$ be defined by (4.1). If there exist non-decreasing functions $h_{1}, h_{2}: \mathbb{R}_{+} \rightarrow \mathbb{R}_{+}$and $\beta_{1}, \beta_{2}>0$ such that

$$
\begin{aligned}
& \|C x-C y\|_{\mathcal{U}} \leq h_{1}\left(\max \left\{\|x\|_{L_{m}^{\infty}},\|y\|_{L_{m}^{\infty}}\right\}\right)\|x-y\|_{L_{m}^{\infty}}^{\beta_{1}}, \quad \text { for all } x, y \in \mathcal{Y}_{\alpha, \tau} \\
& |C y(t)-C y(s)| \leq h_{2}(\|y\| \mathcal{Y})|t-s|^{\beta_{2}}, \quad \text { for all } x, y \in \mathcal{Y}_{\alpha, \tau}, t, s \in[k \tau,(k+1) \tau), k \in \mathbb{N},
\end{aligned}
$$

then $\gamma\left(I-\Phi_{\tau}\right)(r) \leq r^{\beta_{1}} h_{1}(r) \tau^{\alpha}+h_{2}(r) \tau^{\beta_{2}}$. If, in addition, $c_{\infty}<\infty$ where

$$
c_{R}=\max \left\{\sup _{R>r>0} r^{\beta_{1}-1} h_{1}(r), \sup _{R>r>0} h_{2}(r) / r\right\}<\infty,
$$

then $\vec{\delta}\left(C, C_{\tau}\right) \leq c_{\infty} \tau^{\alpha}+c_{\infty} \tau^{\beta_{2}}$

Proof: Let $\tau>0$ and suppose that $C: \mathcal{Y}_{\alpha, \tau} \rightarrow \mathcal{U}$ is causal. By assumptions (5.1) and (5.2)

$$
\begin{aligned}
\left\|C y_{2}-H_{\tau} S_{\tau} C H_{\tau} S_{\tau} y_{2}\right\|_{\mathcal{U}} \leq & \left\|C y_{2}-C H_{\tau} S_{\tau} y_{2}\right\|_{\mathcal{U}}+\left\|C H_{\tau} S_{\tau} y_{2}-H_{\tau} S_{\tau} C H_{\tau} S_{\tau} y_{2}\right\|_{\mathcal{U}} \\
\leq & \left\|C y_{2}-C H_{\tau} S_{\tau} y_{2}\right\|_{\mathcal{U}}+\sup _{\substack{t \in[k \tau,(k+1) \tau) \\
k \geq 0}}\left|\left(C H_{\tau} S_{\tau} y_{2}\right)(t)-\left(C H_{\tau} S_{\tau} y_{2}\right)(k \tau)\right| \\
\leq & h_{1}\left(\max \left\{\left\|y_{2}\right\|,\left\|H_{\tau} S_{\tau} y_{2}\right\|\right\}\right)\left\|y_{2}-H_{\tau} S_{\tau} y_{2}\right\|_{L_{m}^{\infty}}^{\beta_{1}} \\
& +\sup _{\substack{t \in[k \tau,(k+1) \tau) \\
k \geq 0}} h_{2}\left(\left\|H_{\tau} S_{\tau} y_{2}\right\| \mathcal{Y}\right)(t-k \tau)^{\beta_{2}} \\
\leq & h_{1}\left(\left\|y_{2}\right\| \mathcal{Y}\right)\left\|y_{2}\right\|_{\mathcal{Y}}^{\beta_{1}} \tau^{\alpha}+h_{2}\left(\left\|y_{2}\right\| \mathcal{Y}\right) \tau^{\beta_{2}} .
\end{aligned}
$$


Let $r>0$. Then, by the definitions of $\Phi_{\tau}$ and $C_{\tau}$

$$
\gamma\left[I-\Phi_{\tau}\right](r)=\sup _{\substack{\left(y_{2}, C y_{2}\right)^{\top} \in \mathcal{G}_{C} \\\left\|\left(y_{2}, C y_{2}\right)\right\| \mathcal{W} \leq r}}\left\|C y_{2}-H_{\tau} S_{\tau} C H_{\tau} S_{\tau} y_{2}\right\|_{\mathcal{U}} \leq h_{1}(r) r^{\beta_{1}} \tau^{\alpha}+h_{2}(r) \tau^{\beta_{2}}
$$

In the case where $c_{\infty}:=\max \left\{\sup _{r>0} r^{\beta_{1}-1} h_{1}(r), \sup _{r>0} h_{2}(r) / r\right\}<\infty$, then by (5.4), we have

$$
\begin{aligned}
\left\|I-\Phi_{\tau}\right\| & =\sup _{\substack{\left(y_{2}, u_{2}\right)^{\top} \in \mathcal{G}_{C} \\
\left\|\left(y_{2}, u_{2}\right)^{\top}\right\| \neq 0}} \frac{\left\|\left(I-\Phi_{\tau}\right)\left(y_{2}, u_{2}\right)\right\|_{\mathcal{W}}}{\left\|\left(y_{2}, u_{2}\right)^{\top}\right\|_{\mathcal{W}}}=\sup _{\substack{y_{2} \in \mathcal{Y}_{\alpha, \tau} \\
y_{2} \neq 0}} \frac{\left\|\left(0, C y_{2}-H_{\tau} S_{\tau} C H_{\tau} S_{\tau} y_{2}\right)\right\|_{\mathcal{W}}}{\left\|\left(y_{2}, C y_{2}\right)^{\top}\right\|_{\mathcal{W}}} \\
\leq & \sup _{y_{2} \in \mathcal{Y}_{\alpha, \tau}, y_{2} \neq 0} \frac{\left\|C y_{2}-H_{\tau} S_{\tau} C H_{\tau} S_{\tau} y_{2}\right\|_{\mathcal{U}}}{\max \left\{\left\|y_{2}\right\|_{\mathcal{Y}},\left\|C y_{2}\right\|_{\mathcal{U}}\right\}} \leq c_{\infty} \tau^{\alpha}+c_{\infty} \tau^{\beta_{2}} .
\end{aligned}
$$

We remark that, by the definition of $\mathcal{Y}_{\alpha, \tau}$, any $y \in \mathcal{Y}_{\alpha, \tau}$ is such that $|y(t)-y(s)| \leq\|y\| \mathcal{Y}|t-s|^{\alpha}$ for $t, s \in[k \tau,(k+1) \tau)$. Hence any memoryless controller of the form $C y(t)=K\left(y^{r}(t)\right)$ with $K$ Lipschitz satisfies (5.1) and (5.2). Simple examples of dynamic controllers satisfying assumptions (5.1) and (5.2) include cascades of Lipschitz memoryless blocks with stable linear systems, e.g.

$$
u(t)=K\left(\int_{0}^{t} C e^{-A(t-s)} B y(s) d s\right), \quad \text { or } u(t)=\int_{0}^{t} C e^{-A(t-s)} B K(y(s)) d s
$$

where $K: \mathbb{R}^{m} \rightarrow \mathbb{R}^{n}$ is a Lipschitz function and $(A, B, C)$ defines a stable linear system, see Example 5.4 below.

Applying Theorems 2.5 and 2.6, we obtain the following theorems for the respective cases of global and regional stability:

Theorem 5.2. Let $\tau>0$ and let $\mathcal{U}, \mathcal{Y}_{\alpha, \tau}$ be defined as in (3.1)-(3.3). Suppose $P: \mathcal{U}_{a} \rightarrow\left(\mathcal{Y}_{\alpha, \tau}\right)_{a}$ is causal and $C: \mathcal{Y}_{\alpha, \tau} \rightarrow \mathcal{U}$ is an operator satisfying the conditions of Lemma 5.1 for some $h_{1}, h_{2}, \beta_{1}, \beta_{2}$. Suppose that $[P, C]$ is globally well-posed and $\left[P, C_{\tau}\right]$ is locally well-posed.

(i) If $[P, C]$ is globally gain stable and $\tau^{\alpha}+\tau^{\beta_{2}}<c_{\infty}^{-1}\left\|\Pi_{C / / P}\right\|^{-1}$ where $c_{\infty}>0$ is defined by equation (5.3), then $\left[P, C_{\tau}\right]$ is globally well-posed, gain stable and

$$
\left\|\Pi_{C_{\tau} / / P}\right\| \leq\left\|\Pi_{C / / P}\right\| \frac{1+c_{\infty} \tau^{\alpha}+c_{\infty} \tau^{\beta_{2}}}{1-\left(c_{\infty} \tau^{\alpha}+c_{\infty} \tau^{\beta_{2}}\right)\left\|\Pi_{C / / P}\right\|} .
$$

(ii) If $[P, C]$ is gf-stable, and there exists $\varepsilon \in \mathcal{K}_{\infty}$ such that

$$
\left(\gamma\left[\Pi_{C / / P}\right](r)\right)^{\beta_{1}} h_{1}\left(\gamma\left[\Pi_{C / / P}\right](r)\right) \tau^{\alpha}+h_{2}\left(\gamma\left[\Pi_{C / / P}\right](r)\right) \tau^{\beta_{2}} \leq(1+\varepsilon)^{-1}(r) \text { for all } r \geq 0,
$$

then $\left[P, C_{\tau}\right]$ is globally well-posed and gf-stable.

Theorem 5.3. Let $r, \tau>0$ and let $\mathcal{U}, \mathcal{Y}_{\alpha, \tau}$ be defined as in (3.1)-(3.3). Suppose $P: \mathcal{U}_{a} \rightarrow\left(\mathcal{Y}_{\alpha, \tau}\right)_{a}$ is causal and $C: \mathcal{Y}_{\alpha, \tau} \rightarrow \mathcal{U}$ is an operator satisfying the conditions of Lemma 5.1 for some $h_{1}, h_{2}, \beta_{1}, \beta_{2}$. Suppose that $[P, C]$ is globally well-posed on $\mathcal{S}_{r}$ and that $\left[P, C_{\tau}\right]$ is locally well-posed. Also suppose $\left\|\left.\Pi_{C / / P}\right|_{\mathcal{S}_{r}}\right\| \leq p$, (5.1) and (5.2) are satisfied, and that the mapping $\Phi_{\tau}$ defined in (4.1) is such that $\left.\left(\Phi_{\tau}-I\right) \Pi_{C / / P}\right|_{\mathcal{S}_{r}}$ is continuous and compact. If $c_{r}>0$ is defined by $(5.3)$, and $q=c_{r}\left(\tau^{\alpha}+\tau^{\beta_{2}}\right)<p^{-1}$, then $\left[P, C_{\tau}\right]$ is globally well-posed and bounded on $\mathcal{S}_{(1-p q) r}$, and

$$
\left\|\left.\Pi_{C_{\tau} / / P}\right|_{\mathcal{S}_{(1-p q) r}}\right\| \leq\left\|\left.\Pi_{C / / P}\right|_{\mathcal{S}_{r}}\right\| \frac{1+c_{r} \tau^{\alpha}+c_{r} \tau^{\beta}}{1-c_{r}\left(\tau^{\alpha}+\tau^{\beta}\right)\left\|\Pi_{C / / P} \mid \mathcal{S}_{r}\right\|} .
$$


Example 5.4. (A locally stable nonlinear system with a 2-dimensional state) Let $\alpha=n=m=1$ and consider the system when $P, C$ are defined respectively by

$$
\begin{aligned}
& \dot{x}_{1}(t)=x_{1}(t)+x_{2}(t)+u_{1}(t), \quad x_{1}(0)=0, \\
& \dot{x}_{2}(t)=-\sigma x_{2}(t)+x_{1}^{2}(t), \sigma \geq 1, \quad x_{2}(0)=0, \\
& y_{1}(t)=x_{1}(t), \\
& \dot{z}(t)=-\sigma z(t)+y_{2}^{2}(t), \quad z(0)=0 \\
& u_{2}(t)=z(t)-2 y_{2}(t) .
\end{aligned}
$$

For this system, the conditions for Lemma 5.1 are satisfied with $\beta_{1}=\beta_{2}=1, h_{1}(r)=\frac{2}{\sigma} r+2$, $h_{2}(r)=2 r^{2}+2 r$. The closed loop is given by

$$
\dot{x}_{1}(t)=-x_{1}(t)+u_{0}(t)+2 y_{0}(t)+\int_{0}^{t} e^{-\sigma(t-s)}\left(2 y_{0}(s) x_{1}(s)-y_{0}^{2}(s)\right) d s, \quad x_{1}(0)=0,
$$

so the parallel projection $\Pi_{C / / P}$ is the mapping

$$
\left(\begin{array}{c}
y_{0} \\
u_{0}
\end{array}\right) \mapsto\left(\begin{array}{l}
y_{2} \\
u_{2}
\end{array}\right)=\left(\begin{array}{c}
y_{0}(t)-x_{1}(t) \\
\int_{0}^{t} e^{-\sigma(t-s)}\left(y_{0}(s)-x_{1}(s)\right)^{2} d s-2 y_{0}(s)+2 x_{1}(s)
\end{array}\right) .
$$

Multiplying $x_{1}$ to both sides of (5.5) and using the fact $\int_{0}^{t} e^{-\sigma(t-s)} d s \leq \sigma^{-1}$ we obtain

$$
\left|x_{1}(t)\right| \leq \frac{2}{\sigma}\left\|y_{0}\right\| \mathcal{Y} \sup _{s \in[0, t]}\left|x_{1}(s)\right|+\left\|u_{0}\right\|_{\mathcal{U}}+2\left\|y_{0}\right\|_{\mathcal{Y}}+\frac{1}{\sigma}\left\|y_{0}\right\|_{\mathcal{Y}}^{2}
$$

which holds for all $t>0$. Then, for $r<\sigma / 2$ and $\left(y_{0}, u_{0}\right)^{\top} \in S_{r}$, we have

$$
\begin{aligned}
\left\|x_{1}\right\|_{\mathcal{Y}} & \leq\left(1-\frac{2}{\sigma} r\right)^{-1}\left[\left\|u_{0}\right\|_{\mathcal{U}}+2\left\|y_{0}\right\|_{\mathcal{Y}}+\frac{1}{\sigma}\left\|y_{0}\right\|_{\mathcal{Y}}^{2}\right] \leq \frac{3 \sigma r+r^{2}}{\sigma-2 r}, \\
\sup _{\left\|\left(u_{0}, y_{0}\right)^{\top}\right\| \leq r} \frac{\left\|x_{1}\right\|_{\mathcal{Y}}}{\max \left\{\left\|u_{0}\right\|_{\mathcal{U}},\left\|y_{0}\right\|_{\mathcal{Y}\}}\right.} & \leq\left(1-\frac{2}{\sigma} r\right)^{-1}\left(3+\frac{r}{\sigma}\right)=\frac{3 \sigma+r}{\sigma-2 r}
\end{aligned}
$$

and

$$
\begin{aligned}
\left\|\left.\Pi_{C / / P}\right|_{S_{r}}\right\| & =\sup _{\left\|\left(u_{0}, y_{0}\right)^{\top}\right\| \leq r} \frac{\left\|\int_{0}^{t} e^{-\sigma(t-s)}\left(y_{0}(s)-x_{1}(s)\right)^{2} d s\right\|_{\mathcal{U}}+2\left\|y_{0}-x_{1}\right\|_{\mathcal{Y}}}{\max \left\{\left\|u_{0}\right\|_{\mathcal{U}},\left\|y_{0}\right\|_{\mathcal{Y}}\right\}} \\
& \leq \sup _{\left\|\left(u_{0}, y_{0}\right)^{\top}\right\| \leq r} \frac{\left(\left\|y_{0}\right\| \mathcal{Y}+\left\|x_{1}\right\| \mathcal{Y}\right)^{2} \sigma^{-1}+2\left(\left\|y_{0}\right\|_{\mathcal{Y}}+\left\|x_{1}\right\|_{\mathcal{Y}}\right)}{\max \left\{\left\|u_{0}\right\| \mathcal{U},\left\|y_{0}\right\|_{\mathcal{Y}}\right\}} \\
& \leq \frac{(4 \sigma-r)\left(2 \sigma^{2}-r^{2}\right)}{\sigma(\sigma-r)^{2}}=: p .
\end{aligned}
$$

Now,

$$
\Phi_{\tau}:\left(\begin{array}{c}
y \\
C y
\end{array}\right) \rightarrow\left(\begin{array}{c}
y \\
C_{\tau} y
\end{array}\right), \text { for }\left(\begin{array}{c}
y \\
C y
\end{array}\right) \in \mathcal{G}_{C} .
$$

We consider the mappings $\left.\left(I-\Phi_{\tau}\right)\right|_{S_{p r} \cap \mathcal{G}_{C}}$ and $\left.\left(I-\Phi_{\tau}\right) \Pi_{C / / P}\right|_{S_{r}}$. By inequality (5.4),

$$
\left\|\left.\left(I-\Phi_{\tau}\right)\right|_{S_{p r} \cap \mathcal{G}_{C}}\left(\begin{array}{c}
y \\
C y
\end{array}\right)\right\|=\left\|C y-C_{\tau} y\right\| \leq\left(\frac{2}{\sigma}\|y\|_{\mathcal{Y}}^{2}+2\|y\|_{\mathcal{Y}}+2\|y\|_{\mathcal{Y}}^{2}+2\|y\|_{\mathcal{Y}}\right) \tau
$$

which implies

$$
\left\|\left.\left(I-\Phi_{\tau}\right)\right|_{S_{p r} \cap \mathcal{G}_{C}}\right\| \leq\left(4+\frac{5}{2} p r\right) \tau=\left[4+2 r \frac{\left(\sigma_{+} 1\right)(4 \sigma-r)\left(2 \sigma^{2}-r^{2}\right)}{\sigma^{2}(\sigma-2 r)^{2}}\right] \tau=: q .
$$


Since $\left(I-\Phi_{\tau}\right) \Pi_{C / / P}\left(y_{0}, u_{0}\right)^{\top}=\left(0, C y_{2}-C_{\tau} y_{2}\right)^{\top}$, and by (5.4), (5.8), (5.6), (5.7) we obtain

$$
\begin{aligned}
\left\|\left.\left(I-\Phi_{\tau}\right) \Pi_{C / / P}\right|_{S_{r}}\right\| & =\sup _{0 \neq\left(y_{0}, u_{0}\right)^{\top} \in S_{r}} \frac{2(1+1 / \sigma)\left\|y_{2}\right\|_{\mathcal{Y}}^{2}+4\left\|y_{2}\right\|_{\mathcal{Y}}}{\max \left\{\left\|y_{0}\right\| \mathcal{Y},\left\|u_{0}\right\|_{\mathcal{U}}\right\}} \tau \\
& \leq \sup _{0 \neq\left(y_{0}, u_{0}\right)^{\top} \in S_{r}} \frac{2(1+1 / \sigma)\left(\left\|y_{0}\right\|_{\mathcal{Y}}+\left\|x_{1}\right\|_{\mathcal{Y}}\right)^{2}+4\left(\left\|y_{0}\right\|_{\mathcal{Y}}+\left\|x_{1}\right\|_{\mathcal{Y}}\right)}{\max \left\{\left\|y_{0}\right\|_{\mathcal{Y}},\left\|u_{0}\right\|_{\mathcal{U}}\right\}} \tau \\
& \leq \frac{4 \sigma-r}{\sigma-2 r}\left(1+2 \frac{(\sigma+1)\left(4 \sigma^{2}-r^{2}\right)}{\sigma(\sigma-2 r)}\right) \tau .
\end{aligned}
$$

Hence if

$$
\tau<\min \left\{\frac{\sigma-2 r}{4 \sigma-r}\left(1+2 \frac{(\sigma+1)\left(4 \sigma^{2}-r^{2}\right)}{\sigma(\sigma-2 r)}\right)^{-1},\left[4 \frac{\sigma(\sigma-2 r)^{2}}{(4 \sigma-r)\left(2 \sigma^{2}-r^{2}\right)}+2\left(1+\frac{1}{\sigma}\right) r\right]^{-1}\right\}
$$

then $\left\|\left.\left(I-\Phi_{\tau}\right)\right|_{S_{p r} \cap \mathcal{G}_{C}}\right\|<1 / p$ and $\left\|\left.\left(I-\Phi_{\tau}\right) \Pi_{C / / P}\right|_{S_{r}}\right\|<1$.

Finally, we study the continuity and compactness of $\left.\left(I-\Phi_{\tau}\right) \Pi_{C / / P}\right|_{S_{r}}$, equivalently by the continuity and compactness of the mapping

$$
\left(y_{0}, u_{0}\right)^{\top} \mapsto C y_{2}-C_{\tau} y_{2}=C\left(y_{0}-x_{1}\right)-C_{\tau}\left(y_{0}-x_{1}\right) .
$$

By our assumptions and Proposition 3.1 (ii), both $C$ and $C_{\tau}$ are continuous. By (5.5) $\left(y_{0}, u_{0}\right)^{\top} \mapsto x_{1}$ is also continuous. So $\left.\left(I-\Phi_{\tau}\right) \Pi_{C / / P}\right|_{S_{r}}$ is continuous. To establish compactness, let $\left\{\left(y_{0, n}, u_{0, n}\right)^{\top}\right\}$ be a bounded sequence in $\mathcal{S}_{r}$ and $x_{1, n}$ be the solution to (5.5) corresponding to $y_{0, n}, u_{0, n}$. Then $\left\{x_{1, n}\right\}$ is bounded in $L^{\infty}$ and, therefore, from Proposition 4 of [7], it follows that

$$
\left\{x_{1, n}(t)=\int_{0}^{t} e^{-(t-s)}\left[u_{0, n}(s)+2 y_{0, n}(s)+\int_{0}^{s} e^{-\sigma(s-\mu)}\left(2 y_{0, n}(\mu) x_{1, n}(\mu)-y_{0, n}^{2}(\mu)\right) d \mu\right] d s\right\}
$$

has a convergent subsequence, say $\left\{x_{1, n_{i}}\right\}_{i \geq 0}$, in $L^{\infty}$. By (5.5), $\left\{\dot{x}_{1, n_{i}}\right\}_{i \geq 0}$ is also convergent in $L^{\infty}$. Hence $\left\{x_{1, n_{i}}\right\}_{i \geq 0}$ is convergent in $\mathcal{Y}$ and so is $\left\{C\left(x_{0, n_{i}}-x_{1, n_{i}}\right)-C_{\tau}\left(x_{0, n_{i}}-x_{1, n_{i}}\right)\right\}_{i \geq 0}$. This proves the compactness of $\left.\left(I-\Phi_{\tau}\right) \Pi_{C / / P}\right|_{S_{r}}$.

Hence, by Theorem 5.3, $\left[P, C_{\tau}\right]$ is globally well-posed and locally gain stable for small $\tau$ in the sense that $H_{P, C_{\tau}}$ is bounded on $S_{(1-p q) r}$. For example, in the case $\sigma=4, r=0.25$, the system will be locally gain stable for $\tau<0.03497$.

\section{Comments and Conclusions}

In this paper, we investigated the existence of a sampling rate to ensure stability when a continuoustime controller is implemented by a fast sampling and hold strategy. Stability for the sampled-data systems was studied via gap metric approach when the underlying continuous-time closed loop system is known to be stable. The digital controller $C_{\tau}$ was constructed based on sample and hold when the continuous-time controller $C$ is either memoryless or dynamic and stable.

Notice that $\lim _{\tau \rightarrow 0}\left\|\Pi_{C_{\tau} / / P}\right\| \leq\left\|\Pi_{C / / P}\right\|$ in Theorems 4.2, 5.2 and $\lim _{\tau \rightarrow 0}\left\|\left.\Pi_{C_{\tau} / / P}\right|_{\mathcal{S}_{(1-p q) r}}\right\| \leq$ $\left\|\left.\Pi_{C / / P}\right|_{\mathcal{S}_{r}}\right\|$. Analogous bounds can also be obtained for $\left\|\Pi_{P / / C_{\tau}}\right\|$ in Theorems 4.3, 5.3. This shows that the digital controller asymptotically recovers the performance of the underlying continuous-time controller.

We also observe that similar results hold for alternative sampling/holding operations. For example, the holding operator $H_{\tau}$ can be replaced by the 'first order hold' $\hat{H}_{\tau}$ :

$$
\left(\hat{H}_{\tau} z\right)(t)=z_{k-1}+\frac{z_{k}-z_{k-1}}{\tau}(t-k \tau) \text { for any } z=\left(z_{k}\right) \in l^{\infty}, t \in[k \tau,(k+1) \tau) .
$$

Let $\hat{C}_{\tau}$ denote the corresponding digital controller $\hat{C}_{\tau}=\hat{H}_{\tau} C_{d} S_{\tau}$. Then one can prove that the gap between $C_{\tau}$ and $\hat{C}_{\tau}$ decreases to zero as $\tau \rightarrow 0$ become smaller as $\tau$ becomes smaller. Hence analogous fast sampling theorems are also valid for the first order hold. 


\section{References}

[1] Anderson B. D. O., Chongsrid K., Limebeer D.J.N. and Hara S., Direct reduced order discretization of continuous-time controllers, Inter J. Robust Nonlinear Control, 9(1999), 143-155

[2] K. J. Astrom and B. Wittenmark, Computer-controlled systems: theory and design, Pearson Education, 1997

[3] Bian, W. and French, M., Graph topologies, gap metrics and robust stability for nonlinear systems, SIAM J. Contr. Optim. to appear

[4] Cantoni M. and Vinnicombe G., Controller discretisation: a gap metric framwork for analysis and synthesis, IEEE Trans. Auto. Control, 49(2004), 2033-2039

[5] Castillo B., Di Gennaro S., Monaco S. and Normand-Cyrot D., On regulation under sampling, IEEE Trans. Auto. Control, 42(1997), 864-868

[6] Georgiou T. and Smith M. C., Optimal robustness in the gap metric, IEEE Trans. Auto. Control, $35(1990), 673-686$

[7] Georgiou T. and Smith M. C., Robustness analysis of nonlinear feedback systems: an input-output approach, IEEE Trans. Auto. Control, 42(1997), 1200-1221

[8] Georgiou T. and Smith M. C., Robustness of a relaxation oscillator, Int. J. Robust Nonlinear Control, 10(2000), 1005-1024

[9] James M. R., Smith M. C. and Vinnicombe G., Gap metrics, representations and nonlinear robust stability, Preprint

[10] Keller J. P. and Anderson B. D. O., A new approach to the discretization of continuous-time controllers, IEEE Trans. Auto. Control, 37(1992), 214-223

[11] Laila, D.S. Nesic, D. and Teel, A.R. Open and closed loop dissipation inequalities under sampling and controller emulation, Europ. J. Contr. 8(2002), 109-125

[12] Nešić, D. Teel, A. R. and Sontag, E.D., Formulas relating $\mathcal{K} \mathcal{L}$ stability estimates of discrete-time and sampled-data nonlinear systems, Sys. Contr. Lett., 38(1999)49-60

[13] Nešić D. and Laila D. S., A note on input-to-state stabilization for sampled-data systems, IEEE Trans. Auto. Control, 47(2002), 1153-1158

[14] Nešić D., Teel A. R. and Kokotovic P.V., Sufficient conditions for stabilization of sampled-data nonlinear systems via discrete-time approximations, Sys. Contr. Lett., 38(1999), 259-270

[15] Owens D. H., Zheng Y. and Billing S. A., Fast sampling and stability of nonlinear sampled-data systems: Part 1. Existence theorems, IMA J. Math. Control Inf., 7(1990), 1-11

[16] Zheng Y., Owens D. H. and Billing S. A., Fast sampling and stability of nonlinear sampled-data systems: Part 2. Sampling rate estimations, IMA J. Math. Control Inf., 7(1990), 13-33 